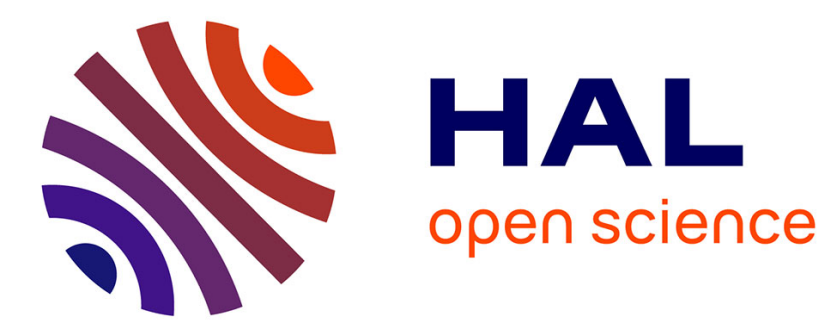

\title{
Les allocataires du RMI inscrits durablement dans le dispositif
}

\author{
Nathalie Blanpain
}

\section{To cite this version:}

Nathalie Blanpain. Les allocataires du RMI inscrits durablement dans le dispositif. Revue des politiques sociales et familiales, 2000, 61, pp.75-83. 10.3406/caf.2000.909 . hal-02149734

\section{HAL Id: hal-02149734 \\ https://hal.science/hal-02149734}

Submitted on 6 Jun 2019

HAL is a multi-disciplinary open access archive for the deposit and dissemination of scientific research documents, whether they are published or not. The documents may come from teaching and research institutions in France or abroad, or from public or private research centers.
L'archive ouverte pluridisciplinaire HAL, est destinée au dépôt et à la diffusion de documents scientifiques de niveau recherche, publiés ou non, émanant des établissements d'enseignement et de recherche français ou étrangers, des laboratoires publics ou privés. 


\section{Les allocataires du RMI inscrits durablement dans le dispositif}

Nathalie Blanpain

\section{Citer ce document / Cite this document :}

Blanpain Nathalie. Les allocataires du RMI inscrits durablement dans le dispositif. In: Recherches et Prévisions, ${ }^{\circ} 61$, 2000. pp. 75-83;

doi : 10.3406/caf.2000.909

http://www.persee.fr/doc/caf_1149-1590_2000_num_61_1_909

Document généré le 09/02/2018 


\title{
Les allocataires du RMI inscrits durablement dans le dispositif
}

\author{
par Nathalie Blanpain \\ INSEE-Division conditions de vie des ménages
}

Le nombre d'allocataires du RMI n'a pas cessé de croître depuis la création du dispositif, passant de 400000 bénéficiaires en décembre 1989 à 1000000 en décembre 1996. Des études font état du caractère passager de l'allocation pour une part non négligeable d'entre eux, attestant que l'objectif d'insertion associé au RMI est atteint au moins en partie. Ainsi, la sortie du dispositif est rapide pour certains : un tiers y reste moins de six mois (Afsa, 1999). Pour d'autres, en revanche, le RMI se pérennise. En décembre 1996, le quart des allocataires sont présents depuis plus de quatre ans et près d'un dixième depuis plus de sept ans (graphique 1).

Une meilleure connaissance des allocataires du RMI inscrits durablement est indispensable à la mise en œuvre de politiques adaptées. L'interrogation, en janvier 1998, d'un échantillon représentatif des allocataires du RMI inscrits dans les caisses d'Allocations familiales (CAF) au 31 décembre 1996 permet d'éclairer leurs situations. On s'intéressera ici à leurs caractéristiques sociodémographiques, leurs conditions de vie, ainsi qu'à leurs perspectives. Un allocataire sera considéré comme inscrit durablement si la date d'ouverture du droit au RMI est antérieure à décembre 1992, ce qui correspond à une présence continue dans le dispositif pendant au moins quatre ans.

\section{Une population âgée et peu diplômée}

Le profil des allocataires du RMI inscrits durablement est, à bien des égards, différent de celui des allocataires entrés récemment. L'âge est notamment un facteur très discriminant: moins de $10 \%$ des bénéficiaires qui se sont inscrits avant 1992 ont moins de 30 ans, contre plus de $40 \%$ de ceux qui se sont inscrits après 1995 (graphique 2). Ces différences reflètent principalement le vieillissement de la population. En effet, les allocataires de moins de 30 ans inscrits durablement sont entrés dans le processus avant 25 ans. Or, c'est un cas rare, puisqu'il faut en l'occurrence être chargé de famille ou attendre un enfant pour prétendre au RMI.

Afin d'éliminer l'effet du vieillissement, il faut retenir l'âge au moment de l'entrée dans le dispositif. Les différences que l'on observait s'estompent nettement. Cependant, quelques effets subsistent : lors de leur inscription, les allocataires de longue durée étaient relativement moins nombreux parmi les moins de 30 ans; ils avaient
Graphique 1 - Répartition des allocataires du RMI selon la date d'ouverture de droit

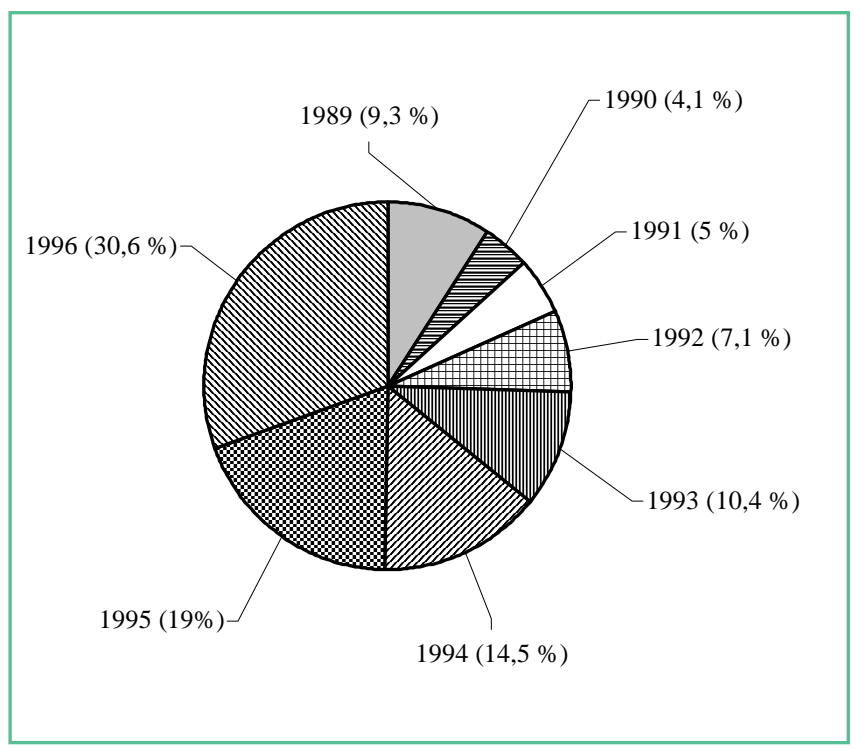

Source : "Revenu minimum d'insertion au 31 décembre 1996 ", Recherches, Prévisions et Statistiques, CNAF, 1997.

Champ : allocataires du RMI inscrits au 31 décembre 1996 dans les CAF de la Métropole.

Graphique 2 - Répartition des allocataires du RMI selon leur âge au 31 décembre 1996 et leur année d'entrée dans le dispositif

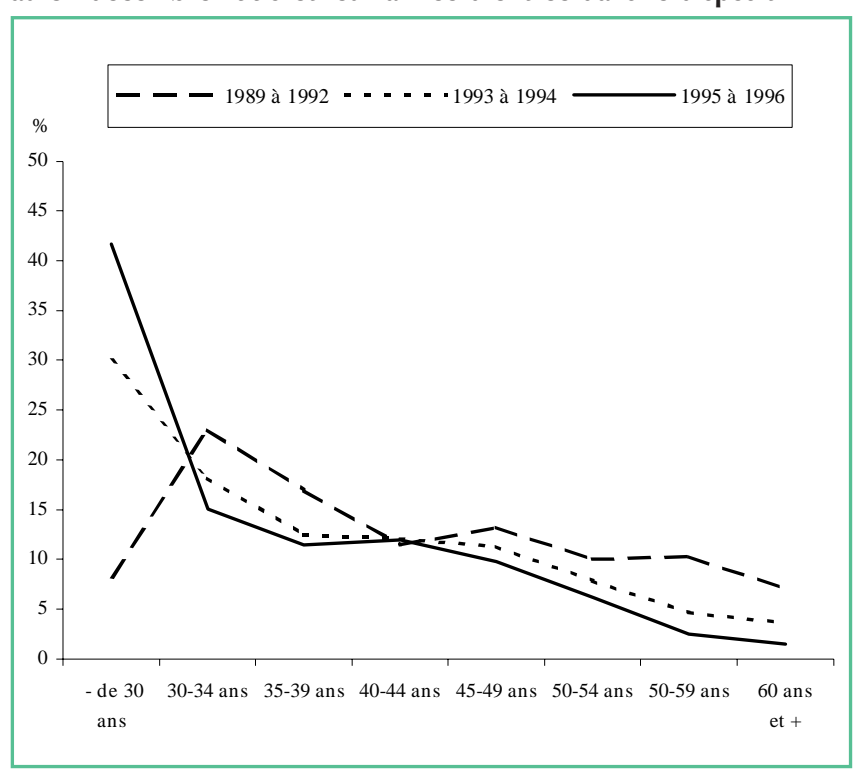

Source : enquête RMI, janvier-février 1998, INSEE, en collaboration avec la CNAF, le CSERC, la DARES, la DIRMI et la DREES.

Champ : allocataires du RMI inscrits au 31 décembre 1996 dans les CAF de Métropole. 


\begin{tabular}{|c|c|c|c|c|c|}
\hline & \multicolumn{5}{|c|}{ Inscrits depuis } \\
\hline & 1989 & 1989-1992 & 1993-1994 & 1995-1996 & Ensemble \\
\hline $\begin{array}{l}\text { Sexe } \\
\text { Homme } \\
\text { Femme }\end{array}$ & $\begin{array}{l}51,3 \\
48,7 \\
\end{array}$ & $\begin{array}{l}52,1 \\
47,9\end{array}$ & $\begin{array}{l}50,5 \\
49,5\end{array}$ & $\begin{array}{l}52,6 \\
47,4\end{array}$ & $\begin{array}{l}51,9 \\
48,1\end{array}$ \\
\hline $\begin{array}{l}\text { Diplôme } \\
\text { Pas de diplôme ou CEP } \\
\text { BEPC, CAP, BEP } \\
\text { Baccalauréat ou plus }\end{array}$ & $\begin{array}{r}68,7 \\
27,6 \\
3,7\end{array}$ & $\begin{array}{l}62,5 \\
27,5 \\
10,0\end{array}$ & $\begin{array}{l}57,1 \\
27,5 \\
15,4\end{array}$ & $\begin{array}{l}45,9 \\
28,3 \\
25,8\end{array}$ & $\begin{array}{l}52,9 \\
27,9 \\
19,2\end{array}$ \\
\hline $\begin{array}{l}\text { Nationalité } \\
\text { Français } \\
\text { Etranger }\end{array}$ & $\begin{array}{l}85,8 \\
14,2 \\
\end{array}$ & $\begin{array}{l}83,9 \\
16,1\end{array}$ & $\begin{array}{l}82,0 \\
18,0\end{array}$ & $\begin{array}{l}84,5 \\
15,5\end{array}$ & $\begin{array}{l}83,7 \\
16,3\end{array}$ \\
\hline $\begin{array}{l}\text { Type de foyer } \\
\text { Allocataire isolé } \\
\text { Parent isolé } \\
\text { Couple sans enfant } \\
\text { Couple avec enfant(s) }\end{array}$ & $\begin{array}{r}65,9 \\
14,3 \\
6,6 \\
13,2 \\
\end{array}$ & $\begin{array}{r}60,4 \\
15,8 \\
6,1 \\
17,7 \\
\end{array}$ & $\begin{array}{r}51,9 \\
22,3 \\
4,8 \\
21,0\end{array}$ & $\begin{array}{r}58,1 \\
19,9 \\
5,2 \\
16,8 \\
\end{array}$ & $\begin{array}{r}57,1 \\
19,5 \\
5,3 \\
18,1\end{array}$ \\
\hline $\begin{array}{l}\text { Catégorie de commune } \\
\text { Commune rurale } \\
\text { Unité urbaine de moins de } 20000 \text { habitants } \\
\text { Unité urbaine de } 20000 \text { à } 100000 \text { habitants } \\
\text { Unité urbaine de plus de } 100000 \text { habitants } \\
\text { Agglomération parisienne }\end{array}$ & $\begin{array}{r}12,9 \\
12,7 \\
19,2 \\
45,4 \\
9,8\end{array}$ & $\begin{array}{l}13,5 \\
13,2 \\
20,4 \\
41,5 \\
11,4\end{array}$ & $\begin{array}{l}14,0 \\
14,3 \\
17,6 \\
41,2 \\
12,9\end{array}$ & $\begin{array}{l}14,4 \\
15,3 \\
17,9 \\
38,0 \\
14,4\end{array}$ & $\begin{array}{l}14,1 \\
14,5 \\
18,4 \\
39,7 \\
13,3\end{array}$ \\
\hline $\begin{array}{l}\text { Situation au cours des mois précédant } \\
\text { la demande de RMI } \\
\text { Travail ou stage } \\
\text { Chômage } \\
\text { Etude ou service militaire } \\
\text { Au foyer ou inactif (y compris longue maladie) } \\
\text { Autre (étranger, ne sait pas...) }\end{array}$ & $\begin{array}{r}27,0 \\
37,8 \\
0,5 \\
25,8 \\
8,9\end{array}$ & $\begin{array}{r}24,4 \\
40,0 \\
1,1 \\
27,0 \\
7,5\end{array}$ & $\begin{array}{r}23,7 \\
44,2 \\
3,0 \\
23,2 \\
5,9\end{array}$ & $\begin{array}{r}22,9 \\
45,6 \\
8,7 \\
17,6 \\
5,2\end{array}$ & $\begin{array}{r}23,5 \\
43,8 \\
5,3 \\
21,4 \\
6,0\end{array}$ \\
\hline $\begin{array}{l}\text { Situation actuelle } \\
\text { Travail ou stage } \\
\text { Chômage } \\
\text { Etude ou service militaire } \\
\text { Au foyer ou inactif (y compris longue maladie) }\end{array}$ & $\begin{array}{r}10,9 \\
50,7 \\
0,8 \\
37,6\end{array}$ & $\begin{array}{r}17,5 \\
49,8 \\
1,1 \\
31,6\end{array}$ & $\begin{array}{r}23,9 \\
54,5 \\
2,1 \\
19,5\end{array}$ & $\begin{array}{r}31,1 \\
51,3 \\
2,7 \\
14,9\end{array}$ & $\begin{array}{r}25,8 \\
51,7 \\
2,2 \\
20,3\end{array}$ \\
\hline $\begin{array}{l}\text { Pour les allocataires qui ne travaillent pas: } \\
\text { recherche d'un emploi } \\
\text { Oui } \\
\text { Non }\end{array}$ & $\begin{array}{l}50,8 \\
49,2\end{array}$ & $\begin{array}{l}53,8 \\
46,2\end{array}$ & $\begin{array}{l}64,0 \\
36,0\end{array}$ & $\begin{array}{l}71,4 \\
28,6\end{array}$ & $\begin{array}{l}64,5 \\
35,5\end{array}$ \\
\hline $\begin{array}{l}\text { Possession du permis de conduire }(1) \\
\text { Oui } \\
\text { Non }\end{array}$ & $\begin{array}{l}45,2 \\
54,8\end{array}$ & $\begin{array}{l}51,2 \\
48,8\end{array}$ & $\begin{array}{l}58,0 \\
42,0\end{array}$ & $\begin{array}{l}64,6 \\
35,4\end{array}$ & $\begin{array}{l}59,6 \\
40,4\end{array}$ \\
\hline $\begin{array}{l}\text { Disposition d'une voiture ou d'un deux-roues } \\
\text { à moteur } \\
\text { Oui } \\
\text { Non }\end{array}$ & $\begin{array}{l}32,2 \\
67,8\end{array}$ & $\begin{array}{l}36,9 \\
63,1\end{array}$ & $\begin{array}{l}42,7 \\
57,3\end{array}$ & $\begin{array}{l}47,7 \\
52,3\end{array}$ & $\begin{array}{l}43,7 \\
56,3\end{array}$ \\
\hline $\begin{array}{l}\text { Signature d'un contrat d'insertion } \\
\text { Oui } \\
\text { Non } \\
\text { Ne sait pas }\end{array}$ & $\begin{array}{r}42,0 \\
54,6 \\
3,4\end{array}$ & $\begin{array}{r}41,1 \\
55,0 \\
3,9\end{array}$ & $\begin{array}{r}40,8 \\
54,6 \\
4,6\end{array}$ & $\begin{array}{r}37,0 \\
59,8 \\
3,2\end{array}$ & $\begin{array}{r}39,0 \\
57,3 \\
3,7\end{array}$ \\
\hline $\begin{array}{l}\text { Perception du RMI en janvier } 1998 \\
\text { Oui } \\
\text { Non }\end{array}$ & $\begin{array}{l}84,8 \\
15,2\end{array}$ & $\begin{array}{l}82,7 \\
17,3\end{array}$ & $\begin{array}{l}76,0 \\
24,0\end{array}$ & $\begin{array}{l}63,0 \\
37,0\end{array}$ & $\begin{array}{l}71,3 \\
28,7\end{array}$ \\
\hline
\end{tabular}

Source: enquête RMI, janvier-février 1998 et septembre-octobre 1998 (pour la possession du permis de conduire uniquement), INSEE en collaboration avec la CNAF, le CSERC, la DARES, la DIRMI et la DREES.

Champ : allocataires du RMI inscrits au 31 décembre 1996 dans les CAF de Métropole.

(1) On a considéréque les personnes sans habitation régulière (1 \% de l'échantillon) ne disposaient pas de voiture.

Lecture du tableau : 52,1 \% des allocataires du champ dont la date d'ouverture de droit au RMI est comprise entre 1989 et 1992 sont des hommes. 
davantage entre 50 ans et 54 ans. Dès leur entrée dans le dispositif, les cohortes de 1989 à 1992 présentaient ces caractéristiques. Par ailleurs, celles-ci ont pu être accentuées par de plus grandes difficultés pour sortir du RMI de la part des plus âgés. En revanche, entre 30 ans et 49 ans, les effets de sélection semblent limités.

Le diplôme est également un autre facteur discriminant. Quel que soit leur âge, les RMIstes de longue durée sont très peu diplômés. Les moins diplômés sortent moins souvent du dispositif. De plus, dès le départ ils étaient particulièrement nombreux.

\section{Des individus plus éloignés du marché de l'emploi lors de leur inscription au RMI}

Au cours des mois précédant leur demande de RMI, $24 \%$ des allocataires inscrits durablement travaillaient ou effectuaient un stage, soit une proportion quasi identique (23\%) à celle des allocataires "récents" (tableau 1). Il s'agissait probablement d'un travail précaire qui n'a pas ou peu donné droit à l'allocation chômage. En effet, la fin du travail a été suivie assez rapidement par la prise en charge du dispositif. II peut également s'agir d'un « effet mémoire »: les allocataires inscrits durablement mentionnent davantage le travail que la période de chômage qui pouvait être relativement courte. On trouve moins souvent de chômeurs ou d'étudiants et plus fréquemment des personnes au foyer ou d'autres inactifs avant leur inscription au RMI. Globalement, les allocataires de longue durée étaient ainsi, lors de leur inscription, plus éloignés du marché du travail.

Les allocataires inscrits durablement ont toutefois des caractéristiques communes aux autres RMIstes. Par exemple, plus de la moitié d'entre eux sont des hommes, ce qui est également le cas pour les allocataires récents (tableau 1). Pour cette variable, les effets de sélection semblent faibles. Toutes choses égales par ailleurs, les femmes sortent autant du dispositif que les hommes, mais il s'agit la plupart du temps de sorties vers des emplois à temps partiel ou de sorties sans emploi (vers une autre allocation par exemple) (Zoyem, 1999).

De même, les différences selon le type de foyer RMI ne sont pas marquées. On remarque, cependant, que les familles monoparentales sont légèrement moins nombreuses parmi les allocataires de longue durée. Les autres écarts sont faibles, de l'ordre de quelques points.

Enfin, comme pour l'ensemble des bénéficiaires du RMI, les personnes de nationalité étrangère représentent environ un sixième des allocataires inscrits durablement dans le dispositif.

\section{La recherche d'emploi}

En janvier 1998, plus d'un allocataire de longue durée sur six travaille, contre près d'un sur trois parmi les allocataires « récents». Parmi les personnes sans emploi, seuls $54 \%$ des « anciens » allocataires déclarent rechercher un emploi, alors que c'est le cas pour $71 \%$ des plus récents. Les raisons peuvent être le découragement au fil du temps, mais aussi l'effet de sélection : ceux qui ne font pas de démarches sortent moins souvent du dispositif (Rioux, 2000).

Parmi les obstacles à la recherche et à la reprise d'un emploi, I'enquête permet notamment de s'intéresser à l'absence de permis de conduire. Seulement la moitié des allocataires inscrits depuis plus de quatre ans le détiennent, contre près des deux tiers de ceux qui sont inscrits depuis moins de deux ans. A âge constant,

\section{Une enquête sur le devenir des bénéficiaires du RMI}

En collaboration avec les principaux organismes chargés de la mise en œuvre de la loi et à leur demande, I'INSEE a réalisé une enquête en trois vagues sur le devenir des bénéficiaires du RMI. Il s'agit principalement de la Délégation interministérielle au RMI (DIRMI), de la Direction de la recherche, des études, de l'évaluation et des statistiques (DREES), de la Caisse nationale des allocations familiales (CNAF), de la direction de l'Animation de la recherche, des études et des statistiques (DARES) du ministère de l'Emploi et de la Solidarité, et du Conseil supérieur de l'emploi, des revenus et des coûts (CSERC). L'échantillon est représentatif des 882047 allocataires du RMI inscrits dans les CAF de Métropole au 31 décembre 1996. Il ne concerne pas les (anciens) agriculteurs inscrits à la Mutualité sociale agricole (21 757 personnes). En septembre et octobre 1997, une première enquête auprès de 10000 allocataires visait principalement à repérer les personnes engagées dans un processus de sortie du RMI. Parmi les 7953 répondants de cette première vague, 3415 personnes ont été interrogées en janvier et février 1998. Plusieurs thèmes ont été abordés dans ce questionnaire: la perception du RMI, le logement, I'occupation principale, l'activité professionnelle, le chômage et la recherche d'emploi, la formation et l'expérience professionnelles, les ressources et les difficultés financières, la santé, les contacts, la sociabilité, les activités non professionnelles. Enfin, une troisième interrogation a eu lieu en septembre et octobre 1998, dans l'objectif de décrire les trajectoires sur une plus longue période. Les résultats présentés dans cet article proviennent principalement de la deuxième vague. Des données de la troisième vague ont été utilisées pour la partie "perspectives». 
Tableau 2 - Le logement et les problèmes de santé des allocataires du RMI

en $\%$

Pour les personnes disposant d'un logement régulier

Nombredepièces du logement

1

2

3

4 et plus

Etes-vous confronté aux problèmes suivants?

Logement trop petit

Oui

Non

Chauffage insuffisant

Oui

Non

Vétusté du logement

Oui

Non

Logementéloigné des transports collectifs

Oui

Non

Logementéloigné des commerces ou équipementscollectifs

Oui

Non

En général, rencontrez-vous des gênes ou des difficultés dans les gestes de la vie quotidienne,

à cause de problèmes de santé ?

Vous avez du malà vous déplacer

Oui

Non

Vous avez du malà vous concentrer

Oui

Non

Vous avez des absences (étourdissements, vertiges)

qui vous interdisent de conduire une voiture

Oui

Non

Depuis un an, avez-vous dû différer une consultation médicale ou un traitement, pour vous ou une personne de votre famille, pour des raisons financières?

Oui

Non

Source : enquête RMI, janvier-février 1998, INSEE en collaboration avec la CNAF, le CSERC, la DARES, la DIRMI et la DREES.

Champ : allocataires du RMI inscrits au 31 décembre 1996 dans les CAF de Métropole.

Lecture du tableau : 14,6 \% des allocataires dont la date d'ouverture de droit au RMI est comprise entre 1989 et 1992 ont un logement d'une pièce. 


\section{L'étude d'un échantillon de stock des allocataires du RMI}

Etudier les allocataires qui font partie du dispositif depuis I'origine à partir d'un échantillon de stock n'est pas simple. En effet, les différences observées en fonction de la date d'inscription dans le dispositif sont, dans la majorité des cas, la conséquence de plusieurs effets que l'on ne sait distinguer. Par exemple, si on suppose que les personnes de l'échantillon inscrites depuis 1989 sont en moins bonne santé que la moyenne, une lecture rapide peut faire penser que leur état de santé s'est dégradé. Mais, ce n'est qu'une hypothèse parmi d'autres. En effet, les entrants de 1989 ont des caractéristiques bien spécifiques: ils sont notamment plus âgés que la moyenne (1) et en moins bonne santé pour cette raison. Ainsi, on observe un moins bon état de santé pour la cohorte de 1989 même en l'absence d'une aggravation de cet état au cours du temps.

De plus, parmi les personnes entrées au RMI en 1989, celles qui souffrent d'un problème de santé restent plus longtemps dans le dispositif. Elles sont donc relativement plus nombreuses dans l'échantillon.

Enfin, au moment de l'interrogation en janvier 1998, seulement une partie des personnes interrogées perçoivent encore le RMI. Les personnes inscrites depuis 1989 sont plus nombreuses dans ce cas. Elles ont par conséquent plus souvent accès à la couverture santé du RMI, ce qui peut influencer leur état de santé. On peut résumer ces quatre effets. Une différence observée en fonction de la date d'inscription peut être due à :

- des effets d'ancienneté : un changement de comportement ou d'état a eu lieu entre l'entrée dans le dispositif et la date de l'enquête. Ce changement peut avoir de multiples causes, comme les effets des politiques du dispositif d'insertion, le découragement des allocataires dans le cas de la recherche d'emploi... Dans l'exemple, l'état de santé s'est dégradé au fil du temps.

Les effets d'ancienneté n'existent pas pour les variables qui restent stables ou relativement stables au cours du temps, comme le diplôme, si l'on considère que les personnes ne sont pas en cours d'études, ce qui est le cas pour les allocataires du RMI (c'est une des conditions d'attribution).

Si on étudie la variable d'âge, cet effet d'ancienneté se manifeste comme le vieillissement de la population. II peut être facilement corrigé en prenant l'âge d'entrée dans le dispositif ;

- des effets de cohortes: les caractéristiques des entrants de 1989 diffèrent de celles des entrants de 1996.

Dans l'exemple, les entrants de 1989 souffrent peutêtre davantage de problèmes de santé dès leur inscription.

Néanmoins, grâce aux données de la CAF, cet effet de cohorte peut être connu au moins pour certaines caractéristiques des entrants (âge, type de famille...) ; - des effets de sélection : les différences observées en fonction de la date d'inscription peuvent caractériser les allocataires qui ne sortent pas du dispositif. En effet, on étudie ici un fichier de stock d'allocataires du
I'écart se réduit, mais subsiste. De même, les bénéficiaires inscrits durablement dans le dispositif disposent moins souvent d'une voiture ou d'un deux-roues à moteur. L'écart est particulièrement flagrant pour les plus âgés d'entre eux. Seulement $26 \%$ des allocataires âgés de 50 ans à 59 ans entrés au RMI entre 1989 et 1992 disposent d'une voiture ou d'un deux-roues à moteur, contre $44 \%$ des allocataires du même âge entrés au RMI après 1995 .

Cet handicap spécifique pour la recherche d'emploi ne se retrouve pas pour l'accès aux transports en commun. Comme pour l'ensemble des allocataires, moins d'un sixième des RMIstes inscrits depuis 1989-1992 déclarent être confrontés à un problème d'éloignement des transports collectifs. Quelle que soit leur ancienneté dans le processus, les RMIstes inscrits dans les CAF habitent rarement une commune rurale (1). Ils sont majoritairement domiciliés dans les grandes villes à l'exception de l'agglomération parisienne, où les loyers demeurent très élevés.

\section{Les conditions de logement}

Curieusement, les conditions de logement ne sont pas vraiment liées à la date d'inscription (tableau 2). On pourrait s'attendre à ce qu'elles soient plus précaires pour les cohortes les plus anciennes: elles pouvaient l'être dès l'entrée au RMI ou ont pu se détériorer au fil du temps. Elles pourraient être également une des caractéristiques des allocataires qui ne sortent pas du dispositif. En fait, il n'y a pas d'écart significatif ni dans le nombre de pièces du logement ni dans la perception personnelle de l'exiguïté, de la vétusté ou de l'éloignement des commerces. Ces caractéristiques ne se modifient guère, sauf en cas de déménagement (2). Par ailleurs, les effets de sélection sont faibles, puisque les personnes qui sortent plus rapidement du dispositif, tels que les jeunes ou les diplômés, ne sont pas particulièrement mieux loties que la moyenne. Il subsiste tout de même des problèmes de chauffage insuffisant un peu plus fréquents pour les bénéficiaires du RMI inscrits depuis longtemps.

\section{Un état de santé dégradé, mais un accès aux soins équivalent}

Quel que soit l'indicateur retenu, les bénéficiaires présents depuis longtemps dans le système souffrent davantage de problèmes de santé. Parce qu'ils sont plus âgés, mais aussi parce que la maladie ou le handicap (1) Les allocataires inscrits dans les régimes agricoles sontexclus du champ del'enquête.

(2) Or, les déménagements sont très probablementsous-estimés en raison des difficultés supplémentaires de recherches que cela entraîne. Pour les mêmes raisons, les personnes ayant des conditions de logement très précaires (centre d'hébergement, caravane, etc.) sontégalementsous-représentées. 
RMI au 31 décembre 1996. On ne connaît donc que la situation des allocataires qui ne sont pas sortis du dispositif à cette date. L'échantillon n'est, en aucun cas, représentatif de I'ensemble des cohortes. II sélectionne, dans chaque cohorte, les personnes qui ont eu les durées les plus longues. Si une variable modifie la probabilité de sortie du dispositif, I'échantillon présente un biais de sélection endogène lorsqu'on étudie cette variable.

Dans l'exemple, la maladie freine la sortie du dispositif. Les personnes en mauvaise santé sont donc surreprésentées dans un échantillon de stock.

Les études utilisant les modèles de durée nous renseignent sur les facteurs qui favorisent la sortie du dispositif et permettent qualitativement de cerner ces effets de sélection ;

- des effets de décalage dus à l'écart temporel entre la date de représentativité de l'échantillon - le 31 décembre 1996 - et le moment de I'interrogation janvier 1998.

Tous les enquêtés étaient allocataires du RMI au 31 décembre 1996. Au moment de l'interrogation, en janvier 1998, certains d'entre eux ne perçoivent plus l'allocation, tandis que d'autres continuent de la percevoir. Si on étudie une variable fortement dépendante du fait d'être ou non encore dans le dispositif (par exemple la couverture maladie), son observation en fonction de l'ancienneté d'entrée au RMI sera perturbée, puisque I'on sait que la sortie du RMI dépend de l'ancienneté.

L'effet de décalage n'est pas forcément lié à la perception de l'allocation; il peut être, par exemple, lié au statut d'occupation.

Dans l'exemple, les personnes inscrites depuis longtemps ont moins souvent retrouvé un travail; elles ont peut être moins souvent déclaré que leur état de santé s'améliorait. Inversement, elles ont moins souvent perdu la couverture santé du RMI et peuvent sans doute se soigner davantage.

Pour éliminer l'effet de la perception du RMI, il faut étudier séparément ceux qui touchent encore I'allocation et ceux qui ne la touchent plus. Parmi les allocataires les plus anciens, ceux qui ne perçoivent plus le RMI en janvier 1998, alors qu'ils le percevaient en décembre 1996, sont peu nombreux. On risque ainsi parfois de se heurter à des problèmes de représentativité de l'échantillon.

Pour les variables qui ne sont pas ou peu influencées à court terme par la sortie du RMI ou la reprise d'un travail (âge, sexe, type de famille, situation avant l'entrée au RMI, signature d'un contrat d'insertion dans le passé, détention du permis de conduire, etc.), la distinction n'est pas nécessaire.

En conclusion, il sera souvent difficile d'expliquer pourquoi on observe des écarts en fonction de la date d'inscription. Néanmoins, il est intéressant de connaître la situation des allocataires inscrits durablement, notamment leurs caractéristiques sociodémographiques et leurs conditions de vie, afin, par exemple, d'adapter les contrats d'insertion qui leur sont proposés.

(1) Allain $\bar{D}$., Les premiers bénéficiaires du RMI : un accès inégal au dispositif d'insertion, Solidarité Santé, Etudes Statistiques, juillet-septembre 1993, $n^{\circ} 3$. freinent la sortie du RMI, les allocataires en mauvaise santé sont en plus grand nombre parmi ceux qui perçoivent I'allocation depuis plusieurs années. Cependant, il ne faut pas négliger une possible dégradation de l'état de santé déclaré, ainsi qu'un état de santé déjà plus fragile au départ.

En revanche, I'accès aux soins ne semble pas dépendre de l'ancienneté. Les allocataires les plus anciens n'ont pas dû reporter plus souvent que la moyenne une consultation médicale pour des raisons financières. En effet, leur couverture maladie est proche de celle des autres allocataires. Si I'on se restreint aux allocataires qui font encore partie du dispositif en janvier 1998, les allocataires de longue durée et les allocataires « récents » ont une couverture santé identique : environ un dixième déclarent être couverts par une mutuelle et les trois quarts par l'aide médicale gratuite (tableau 3).

Parmi les allocataires qui ne font plus partie du dispositif, ceux inscrits depuis plus de quatre ans sont moins souvent couverts par une mutuelle, mais ce déficit est compensé par une plus grande prise en charge à $100 \%$ (aide médicale gratuite en raison de ressources insuffisantes, exonération du ticket modérateur pour des raisons médicales, carte santé encore valide, etc.).

\section{Quatre allocataires sur dix déclarent avoir signé un contrat d'insertion}

Environ $41 \%$ des allocataires qui sont dans le dispositif depuis 1989-1992 ont signé un contrat d'insertion, contre $37 \%$ de ceux qui y sont depuis 1995-1996. Cet écart peut sembler relativement faible. II varie, cependant, à âge donné : il s'élève à quinze points entre 30 ans et 39 ans. Par ailleurs, les effets de sélection sont importants. Les personnes engagées dans un contrat ont plus de chance d'avoir quitté le dispositif, $d^{\prime}$ une part, parce que le contrat peut avoir un réel effet bénéfique en terme de sortie et, d'autre part, parce que les signataires se recrutent souvent parmi «les plus employables ».

Selon Jean-Paul Zoyem, "I'objectif du contrat d'insertion étant de donner des atouts supplémentaires à l'allocataire pour lui permettre de sortir du RMI, on pourrait s'attendreà ce que les moins qualifiés en bénéficient plus souvent. Les résultats de l'enquête montrent le contraire : la probabilité d'avoir signé un contrat croîtfortement avec le niveau d'études (...). On comprend aisément que les allocataires les plus aptes à formuler un projet signent plus souvent un contrat d'insertion. De même de la part du travailleur social, l'incitation à contractualiser sera d'autant plus forte que les chances de réussited'un projetprofessionnel serontélevées » (Zoyem, 1999). 
Tableau 3 - La couverture maladie des allocataires du RMI

en $\%$

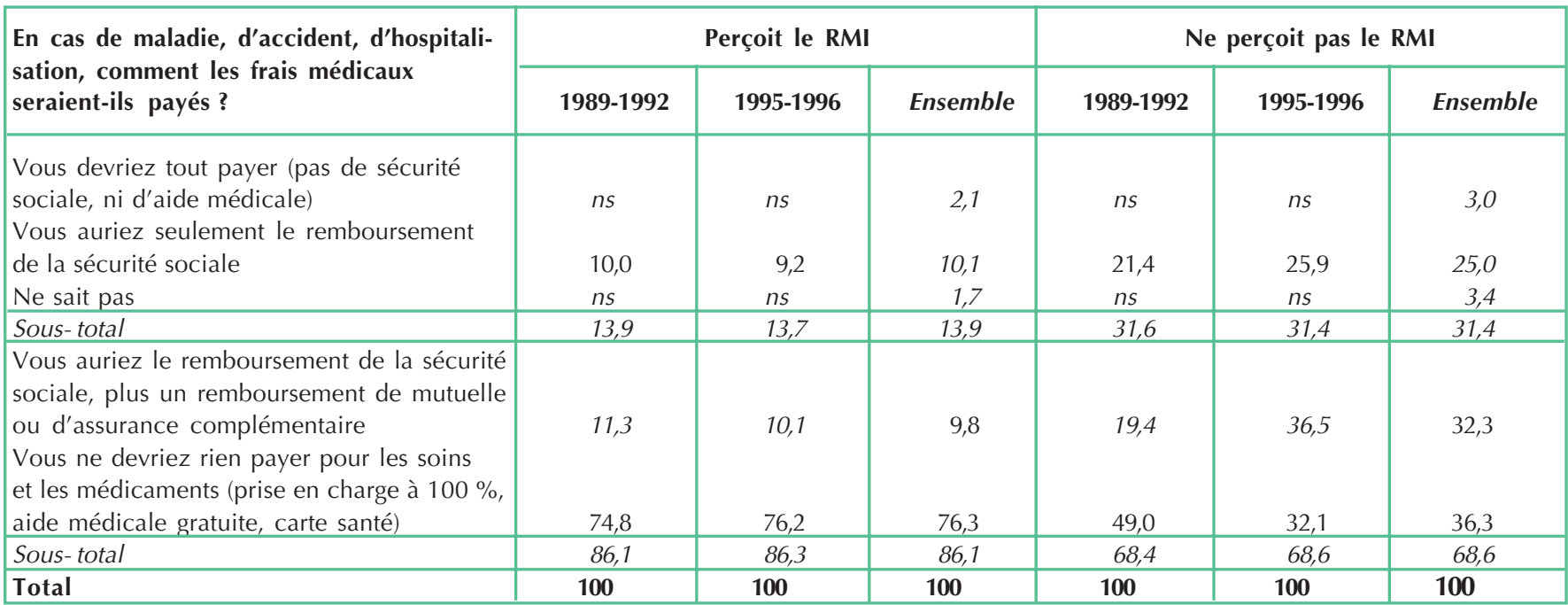

Source : enquête RMI, janvier-février 1998, INSEE en collaboration avec la CNAF, le CSERC, la DARES, la DIRMI et la DREES.

Champ : allocataires du RMI inscrits au 31 décembre 1996 dans les CAF de Métropole.

Lecture du tableau : 10,0 \% des allocataires dont la date d'ouverture de droit au RMI est comprise entre 1989 et 1992 auraient seulement le remboursement de la sécurité sociale, en cas de maladie, d'accidents, d'hospitalisation.

Tableau 4 - Les perspectives de sortie des allocataires du RMI

en $\%$

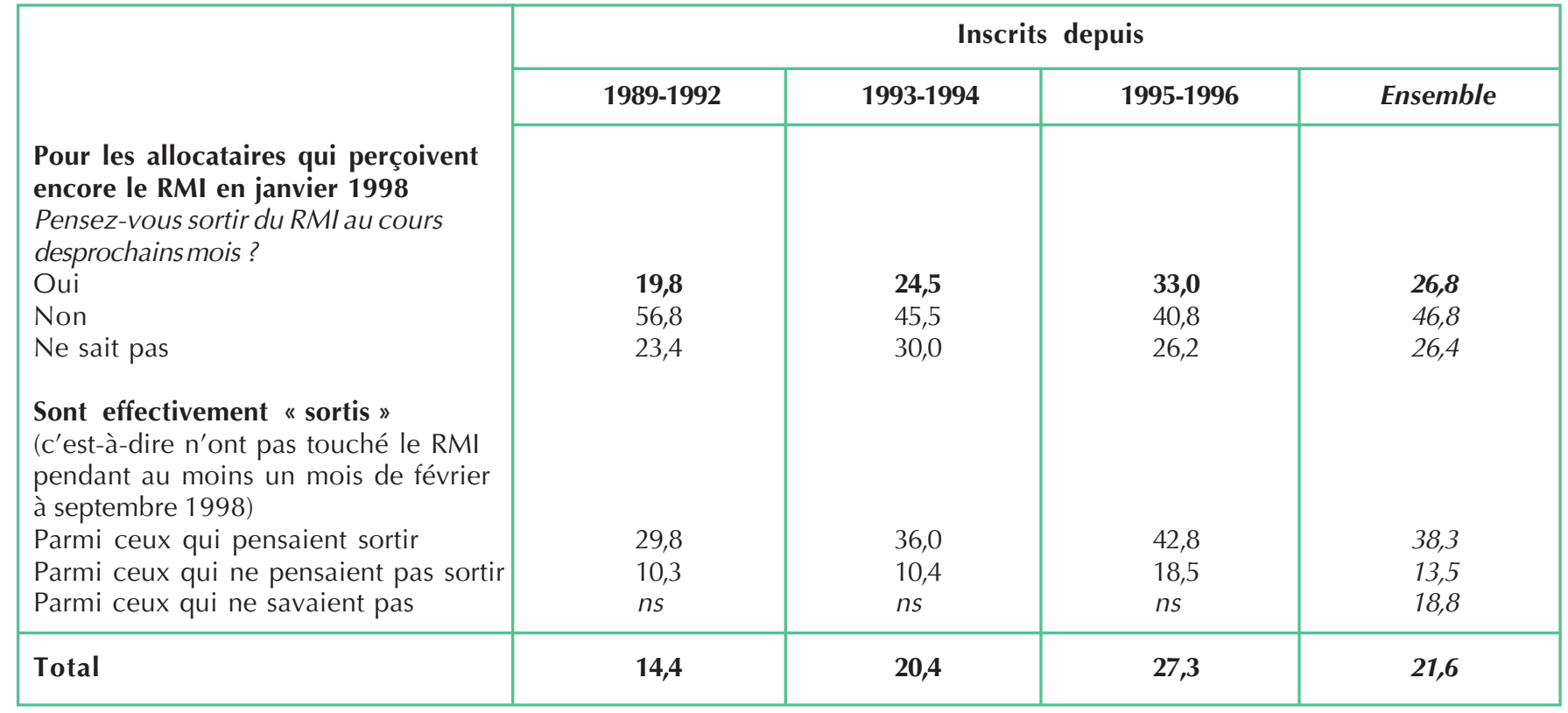

Source : enquête RMI, janvier-février 1998 (pour la réponse à « Pensez-vous sortir du RMI au cours des mois prochains ? ») et septembre-octobre 1998 (pour la réponse à "Sont effectivement sortis »), INSEE en collaboration avec la CNAF, le CSERC, la DARES, la DIRMI et la DREES. Champ : allocataires du RMI inscrits au 31 décembre 1996 dans les CAF de Métropole.

Seuls les allocataires ayant répondu à la deuxième et à la troisième vague de l'enquête (janvier-février 1998 et septembre-octobre 1998) ont été retenus.

Lecture du tableau : en janvier 1998, 19,7 \% des allocataires dont la date d'ouverture de droit au RMI est comprise entre 1989 et 1992 pensaient sortir du RMI au cours des prochains mois. Parmi les allocataires qui pensaient sortir, 29,8\% n'ont effectivement pas touché le RMI pendant au moins un mois de février à septembre 1998. 


\section{Les allocataires du RMI inscrits depuis 1989}

Parmi les allocataires de "longue durée », les allocataires inscrits durablement dès la première année qui a suivi la mise en place du dispositif - 1989 - constituent-ils une population bien spécifique ? On sait que, lors de leur entrée dans le dispositif, leurs caractéristiques sociodémographiques différaient sensiblement de ceux entrés plus tard. Ils étaient notamment plus âgés et moins diplômés. En effet, les personnes qui ont demandé à bénéficier du RMI en 1989 pouvaient être depuis longtemps dans une situation de grande précarité. A l'inverse, les personnes entrées à partir de 1990 étaient probablement depuis peu dans une situation ouvrant droit au RMI.

Dans l'échantillon, on n'observe qu'une partie des cohortes, c'est-à-dire les personnes qui sont restées dans le dispositif au moins jusqu'au 31 décembre 1996. On constate que les caractéristiques déjà observées pour les allocataires de "longue durée » ont plus marquées chez les allocataires inscrits depuis 1989 (tableau 1 et tableau 2). Les effets d'ancienneté et de sélection peuvent aussi expliquer, en partie, le renforcement de ces particularités. Ils sont encore plus âgés, moins diplômés et en moins bonne santé. D'autres effets, qui étaient peu significatifs en incluant les personnes entrées entre 1990 et 1992, apparaissent. Environ $66 \%$ des allocataires inscrits en 1989 sont isolés, contre $58 \%$ des allocataires récents. La vétusté du logement les concerne un peu plus $(28 \%$ contre $23 \%$ ), ainsi que l'éloignement des commerces ou des équipements collectifs (20\% contre $16 \%$ ).
Cependant, on ne peut exclure que des enquêtés aient oublié d'avoir signé ce contrat ou ne l'aient pas identifié parmi les diverses démarches administratives qu'ils ont entreprises depuis leur arrivée au RMI, d'autant plus que l'événement est ancien.

\section{Quelles perspectives?}

Concernant leur sortie du RMI, les allocataires inscrits durablement sont moins optimistes que les allocataires récents. En janvier 1998, un cinquième seulement de ceux inscrits avant 1992 pensent qu'ils ne seront plus au RMI au cours des prochains mois, contre un tiers de ceux entrés en 1995 ou 1996 (tableau 4). A tout âge, leur moindre optimisme est significatif. Les indécis, ceux qui déclarent ne pas savoir, représentent environ un quart des allocataires. Ces prévisions sont-elles en adéquation avec la future réalité ? Il est possible d'en avoir une idée par une nouvelle interrogation de l'échantillon en septembre 1998.

La réalité ne leur donne pas toujours raison. Les allocataires, et particulièrement ceux qui sont dans le dispositif depuis longtemps, envisagent I'avenir de manière plus optimiste qu'il ne le sera. Environ $20 \%$ des allocataires inscrits durablement pensaient, en janvier 1998, sortir au cours des mois suivants. Ils ne seront que $14 \%$ à effectivement ne plus percevoir le RMI pendant au moins un mois de février à septembre 1998 (3).

Si l'on se restreint aux allocataires qui pensaient en sortir, seulement $30 \%$ de ceux inscrits depuis plus de quatre ans ont vu leurs prévisions se réaliser, contre
$43 \%$ de ceux inscrits depuis moins de deux ans. Il est possible qu'une partie des allocataires aient interprété cette question davantage en terme d'espoir qu'en terme de prévision. La probabilité de sortie des allocataires de longue durée étant plus faible, ces derniers voient moins souvent leurs vœux exaucés.

(3) Et encore ne s'agit-il pas toujours de vraies sorties du RMI. La nonperception pendant un mois donné est une définition plus large que celle habituellementretenue.

\section{Références bibliographiques}

Afsa C. et Amira S., Le RMI : un dispositif en mutation, Données sociales, 1999.

Afsa C. et Guillemot D., Plus de la moitiédes sorties du RMI se font grâce à l'emploi, INSEE-première, février 1999, nº 632.

Afsa C., L'insertion professionnelle des bénéficiaires du revenu minimum d'insertion, DREES, Collection Etudes et Statistiques, avril $1999, \mathrm{n}^{\circ} 1$.

Allain D., Les premiers bénéficiaires du RMI : un accès inégal au dispositif d'insertion, Solidarité Santé, Etudes Statistiques, juillet-septembre $1993, n^{\circ} 3$.

INSEE, Le revenu minimum d'insertion, trois ans de mise en œuvre, Economie et Statistique, mars 1992, nº 252.

Rioux L., Les allocataires du RMI : une recherched'emploi active mais qui débouche souventsur un emploi aidé, INSEE-première, juin 2000, $\mathrm{n}^{\circ} 720$.

SESI, "Pauvreté et exclusion », Solidarité Santé, Etudes Statistiques, janvier-mars 1997, $\mathrm{n}^{\circ} 1$.

Zoyem J.-P., Contrat d'insertion et sortie du RMI - Evaluation des effets d'une politique sociale, INSEE, note interne, G9909, juillet 1999. 


\section{Les chances de sortie du RMI}

Effets d'ancienneté et de cohorte : une distinction possible par les données de la CAF

Au moment de l'interrogation en janvier 1998, 83 \% des allocataires de l'échantillon entrés avant 1992 perçoivent encore le RMI, contre seulement $63 \%$ de ceux entrés après 1995. Cette différence s'explique tout d'abord par des effets d'ancienneté. On pourrait penser qu'un allocataire qui est dans le dispositif depuis quatre ans a autant de chances de sortir qu'un allocataire présent depuis cinq ans, l'effet de l'ancienneté disparaissant complètement après un certain nombre d'années. Cela n'est pas le cas. Par exemple, parmi les allocataires entrés dans le dispositif en 1992, $19 \%$ de ceux qui étaient présents depuis quatre ans sont sortis l'année suivante, contre $16 \%$ de ceux qui étaient présents depuis cinq ans (source CAF). Ainsi, pour chaque cohorte, il reste toujours un effet d'ancienneté même s'il s'amenuise d'année en année. Il est possible qu'il devienne quasiment nul après huit ans d'inscription, mais on ne dispose pas encore du recul nécessaire pour le vérifier. L'effet de l'ancienneté sur les sorties vers I'emploi peut être dû à une dégradation des savoir-faire et à une discrimination justifiée ou non des employeurs.

Des effets de cohortes peuvent également expliquer les différences de taux de sortie en fonction de la date d'inscription. On constate une différence significative pendant les premières années d'inscription, tout au moins pour les cohortes anciennes. Un exemple : un an après leur inscription, $20 \%$ des présents en décembre 1989 ne perçoivent plus I'allocation, contre $30 \%$ des présents en décembre 1993 (source CAF). Ces disparités s'expliquent par des profils spécifiques des cohortes, les premiers entrants dans le dispositif étant plus âgés et moins diplômés. Ces effets de cohortes peuvent être perturbés par une conjoncture plus ou moins favorable. Après cinq années d'inscription au RMI, les différences entre toutes les cohortes s'estompent. Il est probable que les personnes les plus " employables » étant sorties du dispositif, les caractéristiques sociodémographiques des cohortes se rapprochent. De plus, même si des caractéristiques plus favorables persistent pour des entrants plus récents, celles-ci s'effacent peut-être devant l'effet stigmatisant du RMI. 